\title{
EDITORIAL
}

\section{How many cath labs do we need?}

\section{Hackett, for the British Cardiac Society Guidelines and Medical Practice Committee}

Heart 2003;89:827-829

\author{
The need for laboratories for cardiac catheterisation \\ and angiography, and pacemaker implantation, is \\ assessed
}

$\mathrm{T}$ he National Service Framework (NSF) for coronary heart disease (CHD) states that: "By international standards, the UK has high rates of CHD but low rates of coronary artery revascularisation. This does not appear to be because most other countries are over-using revascularisation but rather there has been under-provision of revascularisation in the UK. Another major difference between the UK and most other developed countries is that people in the UK wait considerably longer for investigation and treatment than people elsewhere." ${ }^{11}$

The NSF has been welcomed by the cardiology community as a means to improve the medical management of people with coronary heart disease in England. Included among the various objectives and aims of the NSF are plans to increase the number of revascularisation procedures nationally to achieve more than 750 coronary artery bypass graft operations, and more than 750 percutaneous coronary interventional procedures, for each million population per year. Proposed maximum waiting times for investigation and treatment are specified in the NSF. In order to achieve more revascularisation operations, with shorter waiting times, it is obvious that more national capacity for diagnostic cardiac catheterisation and angiography procedures is required.

Historically, the UK has been characterised by fewer numbers of revascularisation procedures, and fewer diagnostic cardiac catheterisation and angiography procedures, when compared with other countries in the European Community. ${ }^{23}$ In various European countries, the number of diagnostic cardiac catheterisation and angiography procedures are usually in the range of 2.0-2.5 (mean 2.2) times the number of revascularisation procedures; and UK planning assumptions for the need for cardiac catheterisation and angiography have previously used a ratio of 2.0 times all cardiac surgical and interventional procedures, including valvar, electrophysiological, and other surgical operations. ${ }^{45}$ For planning purposes it would seem a reasonable surrogate measure to assume that the number of diagnostic cardiac catheterisation and angiography procedures required would be a ratio of 2.2 (upper limit 2.5) times the number of cardiac revascularisation procedures performed or planned.

For medium term planning assumptions, therefore, it should be our aim to provide approximately at least 3300 (upper limit 3750) diagnostic cardiac catheterisation and angiography procedures for each million population per year; and more where the local burden of coronary disease is high.

\section{PACEMAKER IMPLANTATION}

Cardiac catheterisation laboratories are also used for pacemaker implantation, pacemaker lead monitoring, pacemaker lead extraction, cardiac defibrillator implantation, diagnostic and interventional cardiac electrophysiological procedures, as well as for percutaneous coronary interventions. The ideal facility for pacemaker implantation is an operating theatre or dedicated pacing laboratory in which the highest standards of sterility can be maintained; an alternative, but probably less satisfactory for sterile quality, is a shared cardiac catheterisation laboratory. Like other cardiac procedures, the UK has also been characterised by fewer numbers of pacemaker implants when compared with countries in the European Community. ${ }^{67}$ A proposed implant rate of 450 new systems and 100 replacement pacemakers for each million population per year would be comparable with current practice in many European countries, and be a prudent number to use for medium term planning purposes. The National Institute for Clinical Excellence (NICE) has issued guidance on the indications and need for implantable cardiac defibrillators; they have estimated the overall need for new and replacement implantable cardiac defibrillators to be a total of 50 for each million population per year. ${ }^{8}$

The estimates made here of the population need for diagnostic cardiac catheterisation and angiography procedures, pacemaker implantations, and implantable cardiac defibrillators are primarily for cardiac services based in secondary hospital care settings. The British Cardiac Society has published criteria for the provision and use of cardiac catheterisation laboratories in district general hospitals. ${ }^{9}$ The NSF has defined institutional and operator standards for diagnostic cardiac catheterisation and angiography. ${ }^{1}$ It is clear that cardiac catheterisation and angiography procedures can and should be provided in cardiac departments in acute general (secondary care) hospitals serving an appropriate population size. The risks of elective diagnostic cardiac catheterisation and angiography are relatively small, and it can be considered a relatively low risk intervention. ${ }^{10}$ Permanent pacemaker implantation and replacement is also a low risk intervention and appropriate to be performed in acute general hospitals. ${ }^{11}$

No attempt has been made to estimate the population demand or need for pacemaker lead angiographic monitoring, pacemaker lead extraction, diagnostic and interventional cardiac 
electrophysiological procedures (apart from implantable cardiac defibrillators), and percutaneous coronary interventions; the numbers for many of these procedures are rapidly increasing in the UK, and calculations of future need could not currently be projected with ease or confidence. Furthermore, these procedures are generally provided in tertiary care hospital settings, and are not currently or generally provided in most secondary care hospital settings.

\section{LABORATORY REQUIREMENTS}

From the above data, the laboratory requirements for cardiac catheterisation and angiography procedures can be estimated at approximately 700-800 sessions for each million population per year; and for permanent pacemaker and cardiac defibrillator implantations to be approximately 300 sessions. Complete efficiency in use of cardiac laboratories cannot be assumed, and it would be prudent to provide some 15\% excess capacity than the predicted need to allow for the inevitable peaks in demand. ${ }^{12}$ If an excess capacity of $15 \%$ is planned, the estimated annual requirements are approximately 830-950 and 330 sessions, respectively. These figures indicate a weekly need of 17-22 sessions for cardiac catheterisation and angiography procedures, and 6-8 sessions for permanent pacemaker and cardiac defibrillator implantations, for each million population per year.

These data translate into a predicted need to provide:

- one diagnostic cardiac catheterisation and angiography laboratory per 450000 to 600000 population, and

- one dedicated pacemaker and defibrillator implantation laboratory per 1.3 to 1.5 million population, or

- one combined laboratory per 350000 to 400000 population if pacing and cardiac defibrillators are implanted in the same facility as diagnostic cardiac catheterisation and angiography.

These estimated needs will be subject to many other influences and trends, which are difficult to quantify at present. Although there has been a disproportionate decline in the mortality of coronary disease when compared with the incidence in the USA, Canada, Australia, and most western European countries, ${ }^{13-15}$ hospital admissions for coronary disease have actually increased. ${ }^{16}$ Trends suggest that coronary disease is being delayed and increasingly presents in a less severe form, possibly because of modification of coronary risk factors and with modern innovations in treatment. The favourable national trends in risk factors and mortality in the USA and Europe since 1965 have not resulted in much reduction of the national problem of cardiovascular disease. Morbidity and utilisation of health care services remain high. Secondly, on the other hand, factors leading to expected future reductions in coronary heart disease incidence, with fewer expected coronary events, might include secondary and primary prevention measures with statins, prevention associated with Our Healthier Nation initiatives, and an increase in the proportion of people receiving optimal medical treatments. Finally, training requirements-for example, in sessions in which a specialist registrar is being trained-would expect a longer duration of cases. The scheduled caseload might need to be reduced by perhaps $20 \%$ or $25 \%$ for those training sessions.

The need for and provision of laboratories for cardiac catheterisation and angiography and pacemaker implantation should be adjusted for:

- the need for pacemaker lead extraction procedures, diagnostic and interventional cardiac electrophysiological procedures (apart from implantable cardiac defibrillators), and percutaneous coronary interventions, and

- the local burden of disease, and

- the possible reduction in local incidence of coronary morbidity in the future, and
- training requirements, and

- the independent (non-National Health Service) provision and use of local cardiology services.

The Royal College of Physicians and British Cardiac Society have advised that consultant job plans should include a specific number of fixed sessions, and that there should not be more than one session devoted to procedures in a cardiac catheterisation and angiography laboratory for consultants working in a secondary centre. ${ }^{17}$ This would require 24-29 whole time equivalent (WTE) consultant cardiologists per million population to perform cardiac catheterisation and angiography procedures, and pacing and defibrillator implantations, with the range depending on whether cover for leave is provided or not. If each consultant cardiologist in secondary care were to spend an average of 1.5 sessions each week devoted to these laboratory activities, then the range of required numbers of staff to perform these procedures would be 16-19 WTE consultants per million population. The Royal College of Physicians has estimated that the average number of cardiologists in 1997 was 10 per million population (= 1:102 299 population) in England, Wales, and Northern Ireland. ${ }^{17}$ The Fifth report on the provision of services for patients with heart disease recommends an immediate increase in cardiologist staffing to 20 WTE consultants per million population $(=1: 50000)$, and a further increase to 25 WTE per million population $(=1: 40,000)$ by 2010 to deliver modern cardiac services. ${ }^{18}$ Even this staffing level would still remain low when compared with other selected European countries, which have an average of 44 cardiologists per million population $(1: 23000) .{ }^{19}$

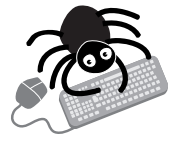

Visit the Heart website and access the supplemental article "How many cathlabs do we need?" posted with the "Fifth report on the provision of services to patients with heart disease" - www.heartjnl.com/ supplemental

\section{REFERENCES}

1 Department of Health. National service framework for coronary heart disease. London: Department of Health. URL: http://www.open.gov.uk/ doh/nsf/coronary.htm

2 Windecker S, Maier-Rudolph W, Bonzel T, et al. Interventional cardiology in Europe 1995. Eur Heart J 1999;20:484-95. http://www.idealibrary.com/links/toc/euhj/20/7/0

3 Maier W, Windecker S, Lablanche JM, et al on behalf of the working group coronary circulation of the European Society of Cardology. Working group report: the European registry of cardiac catheter interventions 1996. Eur Heart J $2001 ; 22: 373-7$. URL: http://www.idealibrary.com/links/doi/10.1053/euhi.2000.2471/pdf 4 Royal College of Physicians of London, Royal College of Surgeons of England. Fourth report of a joint cardiology committee of the Royal College of Physicians of London and the Royal College of Surgeons of England. Provision of services for the diagnosis and treatment of heart disease. Br Heart J 1992;67:106-16

5 British Cardiac Society. A report of a working group of the BCS cardiology in the district hospital. Br Heart J 1994;72:303-8.

6 Ector $\mathbf{H}$, Rickards AF, Linde $C$, et al. The registry of the European working group on cardiac pacing: 1998-1999. Eur Heart J 2001;22 (abstract suppl): 184(abstract P1076)

7 Ector H, Rickards AF, Kappenberger L, et al. The world survey of cardiac pacing and implantable cardioverter defibrillators: calender year 1997Europe. Pace 2001;24:863-8.

8 National Institute for Clinical Excellence. Technology appraisal guidance No 11. Guidance on the use of implantable cardioverter defibrillators for arrhythmias. September 2000. URL: http:// www.nice.org.uk/

9 Council of the British Cardiac Society. Strategic planning for cardiac services and the internal market: role of cardiac catheterization laboratories in district general hospitals. Br Heart J 1994;71:110-2. URL: http://www.bcs.com/publications/pub7.html

10 de Bono D, and the Joint Audit Committee of the British Cardiac Society and the Royal College of Physicians of London. Complications of diagnostic cardiac catheterization: results from 34041 patients in the United Kingdom confidential enquiry into cardiac catheter complications. Br Heart J 1993;70:297-300.

11 Campbell RWF, Charles R, Cowan C, et al. Clinical competence in electrophysiological techniques. Heart 1997;78:403-12. URL: http://www.heart.bmijournals.com/cgi/content/full/78/4/403 
12 Bagust A, Place M, Posnett JW. Dynamics of bed use in accommodating emergency admissions: stochastic simulation model. BM 1999;319:155-8.

13 Rouleau JL, Talasic N, Sussex B, et a 1. Myocardial infarction in patients in the 1990's - their risk factors, stratification and survival in Canada: the Canadian assessment of myocardial infarction (CAMI) study. J Am Coll Cardiol 1996;27:1 11 19-27.

14 Torp-Pedersen C, Hildebrandt P, Køber L, et al. Improving long-term survival of patients with acute myocardial infarction from 1977-1988 in a region of Denmark. Eur Heart J 1995:16:14-20.

15 Uemara K, Piza Z. Trends in cardiovascular disease mortality in industrialized countries since 1950. World Health Stat Q 1988;155-78.
16 National Heart, Lung and Blood Institute. Morbidity and mortality: 1998 chartbook on cardiovascular, lung and blood diseases. Bethesda, Maryland: National Institutes of Health, 1998.

17 Royal College of Physicians. Working for patients. Part 1: a blueprint for effective hospital practice; Part 2: job plans for specialist physicians. June 1999. (Part 1 published in J Roy Coll Physic 1998;32(4):suppl 1).

18 Royal College of Physicians, British Cardiac Society. Fifth report on the provision of services for patients with heart disease. Heart 2002;88/suppl III): iii 7-iii59. URL: http://heart.bmijournals.com/content/vol88/suppl_3

19 Block P, Petch MC, Letouzey JP, on behalf of the Cardiology Monospeciality Section of the UEMS. Manpower in cardiology in Europe. Eur Heart J 2000;21:1 135-40.

\section{IMAGES IN CARDIOLOGY}

\section{Ventricular tachycardia following head injury}

A 24 year old man was admitted to a neurosurgical intensive care unit in a coma following a severe head injury, and ventilated. The patient was noted to have increased ventricular ectopic activity with occasional couplets and salvoes of ventricular tachycardia (VT) subsequently developing more frequent runs of torsades de pointes. Routine biochemistry was normal. Alcohol was detectable in the blood but the concentration was not particularly high. The patient, who was previously fit with no cardiac history or family history of cardiac disease, had a normal echocardiogram. He subsequently developed malignant and very broad sinusoidal VT (panel A). Following unsuccessful initial treatment with intravenous amiodarone which was discontinued, a bolus of magnesium sulfate was given and intermittent overdrive pacing used to stabilise the rhythm. Serum magnesium and calcium concentrations subsequently revealed pronounced hypomagnesaemia and hypocalcaemia. Magnesium infusion was then administered. Serial ECGs with simultaneous magnesium concentrations illustrate atrial fibrillation with widespread repolarisation abnormalities and prolongation of the QTc (panel B). With normalisation of the magnesium concentration there was a resumption of stable sinus rhythm (panel C).

Our patient had developed malignant VT as a consequence of electrolyte imbalance (low magnesium and calcium concentrations) resulting from his head injury. Head injury patients can develop polyuresis following cerebral trauma even without mannitol. Thus they are at high risk of developing hypomagnesaemia, hypocalcaemia, hypophosphataemia, and hypokalaemia because of increased urinary loss - an important consideration in neurosurgical critical care management. The patient made a complete and uncomplicated recovery.

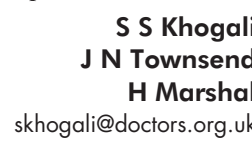

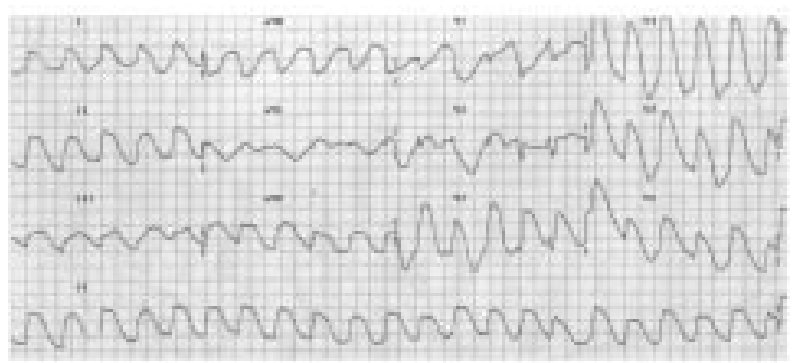

Panel A

$$
\mathrm{Mg}^{2+}=0.61 \mathrm{mmol} / \mathrm{I}
$$$$
\mathrm{Ca}^{2+}=1.78 \mathrm{mmol} / \mathrm{I}
$$

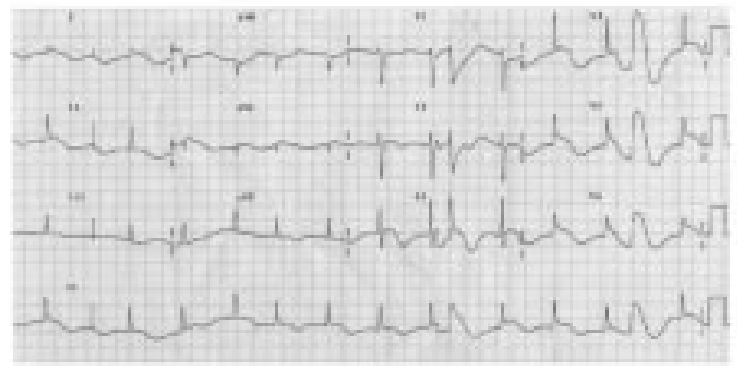

Panel B

$$
\mathrm{Mg}^{2+}=0.77 \mathrm{mmol} / \mathrm{I}
$$$$
\mathrm{Ca}^{2+}=1.69 \mathrm{mmol} / \mathrm{l}
$$

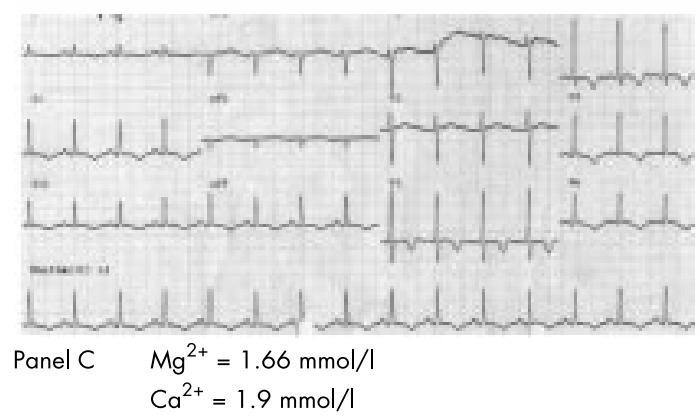

